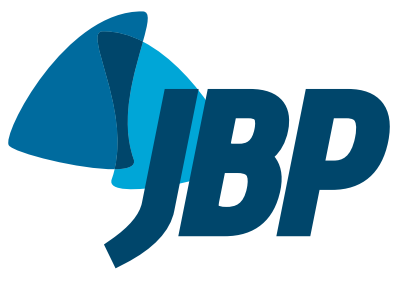

\title{
Response to cytotoxic chemotherapy and overall survival in non-small cell lung cancer patients with positive or negative ERCC1 expression
}

\author{
Helen Naemi Honma,a , Maurício Wesley Perroud Jr ${ }^{1, b}$, \\ André Moreno Morcillo ${ }^{2, c}$, José Vassallo ${ }^{3, d}$, Lair Zambon ${ }^{1, e}$
}

\section{TO THE EDITOR:}

ERCC1 expression levels, as assessed by messenger RNA or protein levels in tumor cells, play a role in the repair of platinum-DNA adducts and influence the response to platinum-based chemotherapy in patients with nonsmall cell lung cancer (NSCLC). ${ }^{(1)}$ Unlike patients with ERCC1-positive NSCLC, patients with ERCC1-negative NSCLC appear to benefit from cytotoxic chemotherapy; however, there is considerable controversy regarding high or low ERCC1 expression levels. (2) This study was aimed at examining the relationship of ERCC1 expression with resistance to chemotherapy with cisplatin plus paclitaxel and global survival. All procedures were performed in accordance with our institutional guidelines, and the study was approved by the local research ethics committee (CEP Protocol no. 550/2009 and CAAE Protocol no. 0442.0.146.0001-09).

Fifty patients with inoperable stage III or IV NSCLC received four cycles of platinum-based chemotherapy (with cisplatin $\left[75 \mathrm{mg} / \mathrm{m}^{2}\right.$ ] or carboplatin [area under the curve, 5] and paclitaxel [200 $\mathrm{mg} / \mathrm{m}^{2}$ ] over a 21-day cycle) at our institution in the period between August of 2009 and June of 2012. Of those 50 patients, only 46 presented with enough tissue for immunohistochemical analysis of ERCC1 expression.

Before initiation of chemotherapy and one month after the end of the fourth cycle, patients underwent CT. Response Evaluation Criteria in Solid Tumors, version 1.1 , were used in order to evaluate patient response to treatment.

Before chemotherapy, bronchoscopic biopsy specimens were analyzed for ERCC1 expression. Tissue sections were deparaffinized and rehydrated. Mouse anti-ERCC1 monoclonal antibody (clone 8F1; Thermo Fisher Scientific, Waltham, MA, USA) was used at 1:200. The primary antibody was visualized with HRP (Agilent Technologies, Inc., Santa Clara, CA, USA) and 3,3'-diaminobenzidine tetrahydrochloride (Agilent Technologies, Inc.). The sections were counterstained with Mayer's hematoxylin.

All immunohistochemical stains were evaluated by the same pathologist, immunoreactivity levels being assessed semiquantitatively under light microscopy. Representative images were acquired at a magnification of $\times 400$ for each specimen. Scores were attributed to the proportion of positive cells (0\%, 0; <25\%, 1; 25-50\%, 2; 50-75\%, 3 ; and $>75 \%, 4$ ) and to staining intensity (no staining, 0 ; weak, 1; moderate, 2; and strong, 3), the final score being obtained by summing the two scores. A final score of 0-2 indicated negative ERCC1 expression, and a final score of 3-7 indicated positive ERCC1 expression.

Statistical analysis was performed with the Statistical Package for the Social Sciences, version 16.0 (SPSS Inc., Chicago, IL, USA). Data are presented as mean \pm standard deviation. Associations between ERCC1 expression and clinicopathological characteristics were evaluated with the chi-square test or Fisher's exact test. The level of significance was set at $p<0.05$. Overall survival was estimated by the Kaplan-Meier method, the log-rank test being used for comparisons. All survival data were analyzed 14 months after data on the last case had been collected.

Patient age ranged from 41 years to 76 years (mean, $61.9 \pm 9.6$ years). Most (65.2\%) of the patients were male, and Whites predominated over non-Whites $(84.8 \%$ vs. $15.2 \%)$. The vast majority of patients had stage IV NSCLC (67.4\%) and a performance status of $0-1$ (65.2\%). In addition, smokers (i.e., current smokers plus former smokers) predominated over never smokers (78.3\% vs. $21.7 \%$ )

Of the NSCLC patients with positive expression of ERCC1, $9(64.3 \%)$ did not respond to platinum-based chemotherapy and 5 (35.7\%) did. As can be seen in Table 1, there were no significant differences between patients with ERCC1-positive NSCLC and those with ERCC1-negative NSCLC regarding response to platinumbased chemotherapy ( $p=0.754)$.

Median survival was 8.88 months for patients with positive expression of ERCC1 and 12.63 months for those with negative expression of ERCC1, showing that ERCC1 expression had no impact on overall survival in the present study ( $p=0.651)$, a finding that is consistent with those of Lee et al. ${ }^{(3)}$ In the present study, there were no significant differences in response to platinum-based chemotherapy or overall survival between NSCLC patients with positive ERCC1 expression and those with negative ERCC1 expression. There is considerable controversy regarding the relationship of ERCC1 expression with response to platinum-based chemotherapy and overall survival. Breen \& Barlési( ${ }^{(4)}$ reported that patients with

1. Departamento de Clínica Médica, Faculdade de Ciências Médicas, Universidade Estadual de Campinas, Campinas (SP) Brasil.

2. Departamento de Pediatria, Faculdade de Ciências Médicas, Universidade Estadual de Campinas, Campinas (SP) Brasil.

3. Departamento de Anatomopatologia, Faculdade de Ciências Médicas, Universidade Estadual de Campinas, Campinas (SP) Brasil.

a. (ID) http://orcid.org/0000.0001.5273-9075; b. (iD) http://orcid.org/0000.0001.5984-9947; c. (D) http://orcid.org/0000.0002.2088-972-x;

d. (iD http://orcid.org/0000.0002.2192-4865; e. (iD) http://orcid.org/0000.0002.3722-1697 
Table 1. Chemotherapy response in patients with positive or negative ERCC1 expression. ${ }^{\text {a }}$

\begin{tabular}{cccccc} 
ERCC1 expression & \multicolumn{2}{c}{ Chemotherapy } & p & OR & $95 \% \mathbf{C l}$ \\
& NRs & Rs & & & \\
Positive & $9(64.3)$ & $5(35.7)$ & 0.754 & 1.23 & $0.34-4.52$ \\
Negative & $19(59.4)$ & $13(40.6)$ & 1.00 & & \\
\hline
\end{tabular}

NRs: nonresponders; and Rs: responders. ${ }^{a}$ Values expressed as $\mathrm{n}(\%)$.

negative ERCC1 expression responded favorably to platinum-based chemotherapy. Simon et al.(5) reported that overall survival was better in patients with high levels of ERCC1 expression than in those with low levels of ERCC1 expression. Lee et al.(6) found a median survival of 7.6 years among patients with high levels of ERCC1 expression and of 4 years among those with low levels of ERCC1 expression $(p=0.046)$. Future studies should employ methods that are more accurate in determining ERCC1 expression levels in order to determine the role of the ERCC1 protein as a predictive biomarker of response to platinum-based chemotherapy.

We would like to thank the thoracic surgery staff of the State University at Campinas for collecting endobronchial biopsy specimens. We would also like to thank Dr. Maurício S. T. Leme and Dr. Aristóteles S. Barbeiro for examining the CT scans. The present study received financial support from the Fundação de Amparo à Pesquisa do Estado de São Paulo (FAPESP, São Paulo Research Foundation; Grant no. 09/52574-5).

\section{REFERENCES}

1. Liao WY, Shih JY, Chang GC, Cheng YK, Yang JC, Chen YM, et al. Genetic polymorphism of XRCC1 Arg399GIn is associated with survival in non-small-cell-lung cancer patients treated with gemcitabine/platinum. J Thorac Oncol. 2012;7(6):973-81. https://doi. org/10.1097/JTO.0b013e31824fe98c

2. Maus MK, Mack PC, Astrow SH, Stephens CL, Zeger GD, Grimminger PP, et al. Histology-related associations of ERCC1, RRM1, and TS biomarkers in patients with non-small-cell lung cancer: implications for therapy. J Thorac Oncol. 2013;8(5):582-6. https://doi. org/10.1097/JTO.0b013e318287c3c5

3. Lee SM, Falzon M, Blackhall F, Spicer J, Nicolson M, Chaudhuri A et al. Randomized Prospective Biomarker Trial of ERCC1 for Comparing Platinum and Nonplatinum Therapy in Advanced Non-Small-Cell Lung Cancer: ERCC1 Trial (ET). J Clin Oncol. 2017;35(4):402-411. https://
doi.org/10.1200/JCO.2016.68.1841

4. Breen D, Barlési F. The place of excision repair cross complementation 1 (ERCC1) in surgically treated non-small cell lung cancer. Eur $J$ Cardiothorac Surg. 2008;33(5):805-11. https://doi.org/10.1016/j. ejcts.2008.01.067

5. Simon GR, Sharma S, Cantor A, Smith P, Bepler G. ERCC1 expression is a predictor of survival in resected patients with non-small cel lung cancer. Chest. 2005;127(3):978-83. https://doi.org/10.1378/ chest.127.3.978

6. Lee KH, Min HS, Han SW, Oh DY, Lee SH, Kim DW, et al. ERCC1 expression by immunohistochemistry and EGFR mutations in resected non-small cell lung cancer. Lung Cancer. 2008;60(3):401-7. https://doi.org/10.1016/j.lungcan.2007.10.014 